\title{
Evaluation of Pregnancy Outcomes among Women with Pregnancies Complicated by Diabetes Mellitus in Abakaliki, South-East, Nigeria
}

\author{
Nwafor Johnbosco Ifunanya*, Onwe Blessing Idzuinya, Obi Vitus Okwuchukwu, \\ Ugoji Darlington-Peter Chibuzor, Ibo Chukwunenye Chukwu, Obi Chuka Nobert, \\ Onuchukwu Victor Jude Uchenna
}

Department of Obstetrics and Gynaecology, Alex Ekwueme Federal University Teaching Hospital, Abakaliki, South-East Nigeria Email: *nwaforjohnbosco97@gmail.com

How to cite this paper: Ifunanya, N.J., Idzuinya, O.B., Okwuchukwu, O.V., Chibuzor, U.D.-P., Chukwu, I.C., Nobert, O.C. and Uchenna, O.V.J. (2019) Evaluation of Pregnancy Outcomes among Women with Pregnancies Complicated by Diabetes Mellitus in Abakaliki, South-East, Nigeria. Journal of Diabetes Mellitus, 9, 69-76. https://doi.org/10.4236/jdm.2019.93008

Received: July 13, 2019

Accepted: August 9, 2019

Published: August 12, 2019

Copyright $\odot 2019$ by author(s) and Scientific Research Publishing Inc. This work is licensed under the Creative Commons Attribution International License (CC BY 4.0).

http://creativecommons.org/licenses/by/4.0/

(c) () Open Access

\begin{abstract}
Background: Pregnancies complicated by diabetes are associated with significant increase in maternal and perinatal morbidity and mortality. The management of diabetes in pregnancy is a great challenge in a low resource setting because of limited resources and facilities to care for these women. Aim: To determine the maternal and perinatal outcomes of diabetic pregnant women managed at Alex Ekwueme Federal University Teaching Hospital Abakaliki, Southeast, Nigeria. Materials and methods: This was a 7-year retrospective case-control study that compared pregnancy outcomes among women with pregnancies complicated by diabetes and those without diabetes managed at Alex Ekwueme Federal University Teaching Hospital, Abakalikibetween January $1^{\text {st }}, 2012$ and December $31^{\text {st }}$, 2018. The statistical analysis was done using SPSS version 22. Results: The incidence of diabetes in pregnancy in this study was 6.6 per 1000 deliveries. Of 126 women in diabetic arm of the study, 81 were diagnosed during pregnancy and 45 were known diabetic prior to conception. Over two-thirds of 126 women with pregnancy complicated by diabetes achieved good blood glucose control during pregnancy. Both groups differ in their mean BMI and women with diabetes in pregnancy were more likely to be obese compared with control (diabetic; 30.1 \pm 2.5 versus control; $23.4 \pm 2.1, \mathrm{P}<0.0001$ ). Pregnant women with diabetes were more likely to be delivered by cesarean section when compared with non-diabetic women (86 versus $23, \mathrm{OR}=9.6,95 \%$ CI: 5.35 - 17.32, $\mathrm{P}<0.0001$ ). Similarly, the incidence of polyhydramnious was higher in paturients with diabetes when compared with the control groups (26 versus $13, \mathrm{OR}=2.2$, $95 \%$ CI: $1.10-4.63, \mathrm{P}=0.02)$. There were no differences between both study
\end{abstract}


groups with regards to other maternal outcomes. The incidence of fetal macrosomia, neonatal hypoglycemia and neonatal respiratory distress syndrome were significantly higher among women whose pregnancies were complicated by diabetes when compared with the control [Diabetics; fetal macrosomia (62.7\%), neonatal hypoglycemia (44.4\%) and neonatal respiratory distress syndrome (22.2\%) versus Control; fetal macrosomia (34.1\%), neonatal hypoglycemia $(7.9 \%)$ and neonatal respiratory distress syndrome (5.6\%) respectively]. Conclusion: Women with pregnancies complicated by diabetes had a higher incidence of adverse maternal and perinatal outcomes. Clinical recognition of diabetes in pregnancy is important because institution of therapy, and antepartum fetal surveillance can reduce the maternal and perinatal morbidity and mortality associated with the condition.

\section{Keywords}

Pregnancies, Complicated, Diabetes Mellitus, Maternal, Perinatal, Abakaliki

\section{Introduction}

Diabetes mellitus (DM) is a common medical complication of pregnancy and it is associated with adverse maternal and perinatal outcomes [1].

The prevalence of diabetes worldwide doubled since 1980 and the rate in the general population paralleled the rate in pregnancy [1]. It complicates about $10 \%$ of all pregnancies globally [2]. Diabetes during pregnancy can be divided into two subtypes: pregestational diabetes mellitus (PDM) type 1 (T1DM) or type 2 (T2DM), and gestational diabetes mellitus (GDM) [3]. GDM is defined as diabetes with first onset during pregnancy [3]. GDM usually constitutes around $90 \%$ of all pregnancies complicated by diabetes, while pre-existing diabetes accounts for the remaining $10 \%$ [4].

Pregnancies complicated by diabetes are associated with significant increase in maternal and perinatal risks [5]. Concerning pregnant women with diabetes, the risks of pre-eclampsia, cesarean section and maternal mortality are significantly higher than in mothers without diabetes [5]. Fetuses of women with diabetes in pregnancy have increased risk for malformations, especially congenital heart diseases and anomalies of the nervous system [6]. This is due to poor glycaemic control during the period of organogenesis which occurs in the first trimester of pregnancy and it is believed to be due to the negative effects of the hyperglycemic environment on the developing fetus [7] [8]. To reduce adverse maternal and perinatal outcomes, it is essential that glucose levels are maintained at optimal level, and to achieve normal glucose level, good compliance to therapy and astute glucose monitoring are required [9] [10]. Therefore, it is important to examine the outcomes of care for women with pregnancy complicated with diabetes in our hospital and to assess if the care provided is sufficient or needs to be changed. The aim of this study was to determine the maternal and perinatal outcomes of diabetic pregnant women managed at Alex Ekwueme Federal Uni- 
versity Teaching Hospital Abakaliki, Southeast, Nigeria.

\section{Materials and Method}

Study area: Abakaliki is the capital of Ebonyi state which is located in the south-east geographical zone of Nigeria. Alex Ekwueme Federal University Teaching Hospital (formerly known as Federal Teaching Hospital) is a tertiary institution located in Abakaliki. The hospital receives referral from all parts of the state and neighboring states of Benue, Enugu, Cross River and Abia as well as any part of the country.

Study design: This was a 7-year retrospective case-control study undertaken between January $1^{\text {st }}, 2012$ and December $31^{\text {st }}, 2018$ at the Department of Obstetrics and Gynaecology of Alex Ekwueme Federal University Hospital, Abakaliki. The cases were women with pregnancies complicated by diabetes (Type 1 Diabetes Mellitus, Type 2 Diabetes Mellitus and Gestational Diabetes). The study controls were pregnant women without diabetes. The exclusion criteria were multiple pregnancies and incomplete or unavailable medical records. In this study adequate glucose control was defined as Fasting blood glucose $<95 \mathrm{mg} / \mathrm{dl}$, and/or 1-hour postprandrial blood glucose $<140 \mathrm{mg} / \mathrm{dl}$ and/or 2-hour blood glucose $<120 \mathrm{mg} / \mathrm{dl}[10]$. Women with blood glucose above these ranges were adjudged to have poor blood glucose control [10].

Data collection: The hospital numbers of the eligible women during the study period were retrieved from the admission register in the Antenatal ward. Then, the case notes were retrieved from the Medical Records Department of the hospital using the hospital numbers. A proforma containing information on the maternal age, parity, gestational age at delivery, Body mass index (BMI), and blood glucose measurements during prenatal period were used to extract information from the case notes. Data regarding risk factors for diabetes and pregnancy outcomes were also noted. The pregnancy outcomes were compared between pregnant women with and without diabetes. The approval for the study was sought for and obtained from the Research and Ethics Committee of the Alex Ekwueme Federal University Teaching Hospital, Abakaliki.

Statistical analysis: The data was entered and analyzed using SPSS Version 22.0 (SPSS Inc., Chicago, IL, USA). Data are presented as mean (standard deviation) or as a percentage with range, as appropriate. Odds ratios (OR) with 95\% CIs were used to identify factors associated with maternal and perinatal outcomes. Multivariate logistic regression was used to control for confounding factors. A p-value $<0.05$ was considered significant.

\section{Results}

There were 23,450 deliveries during the study period of which 154 were women with diabetes in pregnancy. The incidence of diabetes in pregnancy in this study was 6.6 per 1000 deliveries. Twenty-eight women were excluded from the analysis due to multiple gestation (20) and incomplete record (8). The cases were 126 
women with pregnancy complicated by diabetes, whereas the control group were 126 randomly selected women without diabetes with a complete record to match the diabetic group in terms of age and parity.

Table 1 shows the socio-demographic and clinical characteristics of the study cohorts. The age distribution of the women ranged from 15 to 45 years. The majority of the women were between 35 and 45 years in the diabetic arm and between 25 - 34 years in the control group. There were no significant differences in the mean age of women with diabetic and the non-diabetic group (diabetic; $26.1 \pm 4.1$ versus control; $25.2 \pm 3.9, \mathrm{P}=0.08)$. Grandmultiparas accounted for majority of the study participants in diabetic (46.1\%) and control $(40.5 \%)$ groups. There were no significant differences in mean parity between both study groups (diabetic; $3.2 \pm 1.4$ versus control; $2.9 \pm 1.6, \mathrm{P}=0.11$ ). Both groups differ in their mean BMI and women with diabetes in pregnancy were more likely to be obese compared with control (diabetic; $30.1 \pm 2.5$ versus control; $23.4 \pm 2.1$, P $<0.0001)$. Also diabetic pregnant women were delivered at earlier gestational age when compared with non-diabetic controls (diabetic; $38.2 \pm 1.2$ versus control; $39.6 \pm 1.3, \mathrm{P}<0.0001)$. Of 126 women in diabetic arm of the study, 81 were diagnosed during pregnancy and 45 were known diabetic prior to conception. Over two-third of 126 women with pregnancy complicated by diabetes achieved good blood glucose control during pregnancy.

The comparison of risk factors for diabetes in pregnancy among women with and without pregnancies complicated by diabetes is shown in Table 2. The significant risk factors found among diabetic paturients were family history of diabetes, obesity (BMI $>30 \mathrm{~kg} / \mathrm{m}^{2}$ ), history of macrosomia (wt $>4 \mathrm{~kg}$ ), recurrent glycosuria, unexplained recurrent $>2$ miscarriages and history of polyhydramnious.

Table 3 shows maternal and perinatal outcomes in diabetics and control group.

Table 1. Socio-demographic and clinical characteristics of the diabetics and control group.

\begin{tabular}{cccc}
\hline Characteristic & $\begin{array}{c}\text { Diabetic group } \\
(\mathbf{N = 1 2 6 )}\end{array}$ & $\begin{array}{c}\text { Control group } \\
(\mathbf{N}=126)\end{array}$ & P-value \\
\hline Age (years) & & & \\
$15-24$ & $12(9.5 \%)$ & $18(14.3 \%)$ & - \\
$25-34$ & $48(38.1 \%)$ & $56(44.4 \%)$ & - \\
$35-45$ & $66(52.4 \%)$ & $52(41.3 \%)$ & 0.08 \\
Mean age & $26.1 \pm 4.1$ & $25.2 \pm 3.9$ & - \\
Parity & & & - \\
$0-2$ & $12(9.5 \%)$ & $25(19.8 \%)$ & 0.11 \\
$3-5$ & $56(44.4 \%)$ & $50(39.7 \%)$ & $<0.0001$ \\
$>5$ & $58(46.1 \%)$ & $51(40.5 \%)$ & - \\
Mean parity & $3.2 \pm 1.4$ & $2.9 \pm 1.6$ & - \\
Mean BMI (kg/m $\left.{ }^{2}\right)$ & $30.1 \pm 2.5$ & $23.4 \pm 2.1$ & - \\
Mean Gestational age at delivery (weeks) & $38.2 \pm 1.2$ & $39.6 \pm 1.3$ & - \\
Pregestational diabetic & $45(35.7 \%)$ & - & - \\
Gestational diabetic & $81(64.3 \%)$ & - & - \\
Adequate blood glucose control & $86(68.3 \%)$ & & - \\
Poor blood glucose control & $40(31.7 \%)$ & & - \\
\hline
\end{tabular}


Table 2. Risk factors for diabetes mellitus in pregnancy.

\begin{tabular}{|c|c|c|c|c|}
\hline Risk factor & $\begin{array}{c}\text { Diabetic group } \\
(\mathrm{N}=126)(\%)\end{array}$ & $\begin{array}{l}\text { Control group } \\
(\mathrm{N}=126)(\%)\end{array}$ & OR $(95 \% \mathrm{CI})$ & P-value \\
\hline Age $>30$ years & $72(57.1)$ & $61(48.4)$ & $1.4(0.86-2.33)$ & 0.16 \\
\hline Family history of diabetes & $86(68.3)$ & $23(18.3)$ & $9.6(5.35-17.32)$ & $<0.0001$ \\
\hline Obesity $\left(\mathrm{BMI}>30 \mathrm{~kg} / \mathrm{m}^{2}\right)$ & $79(62.7)$ & $14(11.1)$ & $13.4(6.93-26.09)$ & $<0.0001$ \\
\hline History of macrosomia (wt $>4 \mathrm{~kg}$ ) & $67(53.2)$ & $23(18.3)$ & $5.1(2.87-9.01)$ & $<0.0001$ \\
\hline Recurrent glycosuria & $80(63.5)$ & $8(6.3)$ & $25.7(11.5-57.24)$ & $<0.0001$ \\
\hline Previous unexplained neonatal death & $12(9.5)$ & $0(0)$ & - & - \\
\hline Unexplained recurrent $>2$ miscarriages & $15(11.9)$ & $4(3.2)$ & $4.1(1.33-12.79)$ & 0.009 \\
\hline Previous congenital malformations & $10(7.9)$ & $7(5.6)$ & $1.5(0.54-3.98)$ & 0.45 \\
\hline History of polyhydramnious & $23(18.3)$ & $5(3.9)$ & $5.4(1.98-14.72)$ & 0.0003 \\
\hline History of stillbirth & $14(11.1)$ & $6(4.8)$ & $2.5(0.93-6.73)$ & 0.06 \\
\hline
\end{tabular}

Table 3. Maternal and perinatal outcomes in diabetics and control group.

\begin{tabular}{ccccc}
\hline Outcome & Diabetic group Control group & OR (95\%CI) & P-value \\
& $(\mathrm{N}=126)(\%)$ & $(\mathrm{N}=126)(\%)$ & & \\
\hline Caesarean section & $86(68.3)$ & $23(18.3)$ & $9.6(5.35-17.32)$ & $<0.0001$ \\
Preeclampsia & $34(26.9)$ & $28(22.2)$ & $1.3(0.73-2.29)$ & 0.38 \\
Polyhydramnious & $26(20.6)$ & $13(10.3)$ & $2.2(1.10-4.63)$ & 0.02 \\
Shoulder dystocia & $12(9.5)$ & $8(6.3)$ & $1.6(0.61-3.94)$ & 0.35 \\
Preterm delivery & $23(18.3)$ & $19(15.1)$ & $1.2(0.65-2.45)$ & 0.49 \\
Congenital malformation & $14(11.1)$ & $8(6.3)$ & $1.8(0.74-4.56)$ & 0.18 \\
Stillbirth & $32(25.4)$ & $21(16.7)$ & $1.7(0.92-3.15)$ & 0.09 \\
Early neonatal death & $8(6.3)$ & $6(4.8)$ & $1.4(0.46-4.03)$ & 0.58 \\
Macrosomia & $79(62.7)$ & $43(34.1)$ & $3.2(1.94-5.43)$ & $<0.0001$ \\
APGAR score $<7$ at 5th min & $15(11.9)$ & $14(11.1)$ & $1.1(0.49-2.34)$ & 0.84 \\
Neonatal hypoglycemia & $56(44.4)$ & $10(7.9)$ & $9.3(4.45-19.36)$ & $<0.0001$ \\
Neonatal respiratory distress syndrome & $28(22.2)$ & $7(5.6)$ & $4.9(2.03-11.59)$ & 0.0001 \\
\hline
\end{tabular}

Pregnant women with diabetes were more likely to be delivered by caesarean section when compared with non-diabetic women (86 versus 23 , OR $=9.6$, 95\%CI: 5.35 - 17.32, P $<0.0001)$. Similarly, the incidence of polyhydramnious was higher in paturients with diabetes when compared with the control groups (26 versus $13, \mathrm{OR}=2.2,95 \% \mathrm{CI}: 1.10-4.63, \mathrm{P}=0.02$ ). There were no differences between both study groups with regards to other maternal outcomes. The incidence of fetal macrosomia, neonatal hypoglycemia and neonatal respiratory distress syndrome were significantly higher among women whose pregnancies were complicated by diabetes when compared with the control. There were no differences in other perinatal outcomes in both groups.

\section{Discussion}

In this study we evaluated maternal and fetal outcomes and predisposing factors in all pregnancies complicated by diabetes from 2012 to 2018 at Alex Ekwueme Federal University Teaching Hospital, Abakaliki, Southeast, Nigeria. For comparison, a group of pregnancies not complicated by diabetes matched for age, parity and date of delivery, was investigated. We found that the incidence of diabetes in pregnancy was 6.6 per 1000 deliveries $(0.66 \%)$. This finding was lower 
than the values that were reported in other African countries [11] [12] [13]. A systematic review of six countries in Africa estimated the overall prevalence of GDM to be 5\%; however, there is variability due to the availability of screening procedures and diagnostic criteria [14]. Two recent studies indicated a $2.9 \%$ prevalence rate in Kenya, and a 9.1\% rate in South Africa. [14] Both studies used the WHO (2013) criteria based on the International Association of the Diabetes and Pregnancy Study Groups (IADPSG) evidenced-based practice recommendations.

This study showed that pregnant women with diabetes had significantly higher mean early pregnancy BMI when compared with those without diabetes. Diabetes in pregnancy has been observed to be linked with high maternal BMI. Studies have shown that the risk was increased in both overweight and obese mothers. In this study, $62.7 \%$ of the women with pregnancies complicated by diabetes were obese with $B M I \geq 30 \mathrm{~kg} / \mathrm{m}^{2}$, whereas the control group without diabetes had significantly lower mean BMI in early pregnancy so primary prevention of obesity is important in preventing development of diabetes in pregnancy and its associated pregnancy complications.

The recognized risk factors associated with increased risk for diabetes in pregnancy which was largely derived from populations of European extraction appear to be applicable for pregnant women in Abakaliki. This study supports this finding for our women, with age, BMI, family history, previous history of macrosomia and glycosuria all being significant. The best approach to screen for GDM remains a controversy. Many sources, including the American Diabetes Association, recommended universal screening in the past, but selective screening is more cost-effective in low-prevalence populations [15] [16] [17]. Universal screening remains the approach of choice in populations with a moderate or high prevalence of GDM.

In the present study the mode of delivery was caesarean section in $68.3 \%$ of the women with diabetes in comparison to only $18.3 \%$ of the women without diabetes. As all pregnancies complicated by diabetes are followed more frequently with ultrasound, and if the fetal growth is considered abnormally increased a caesarean section is scheduled at 39 weeks gestation. The increased rate of caesarean section was in accordance with the findings of increased rates of CS in all pregnancies complicated by diabetes found in a retrospective study from Israel and Sweden [16] [17].

The risk for fetal macrosomia was higher in diabetic pregnancies compared to the pregnancies without diabetes [17]. This is not surprising as $31.7 \%$ of women with diabetes in this study had poor glycaemic control at the time of delivery. Level of glycemic control is considered to be the most important risk factor for congenital malformations, stillbirth and early neonatal death. In contrast to other studies [14] [15], we found no difference when these outcomes were compared among women with and without diabetes in pregnancy.

Clinical recognition of diabetes in pregnancy is important because therapy, 
including nutrition therapy, insulin when necessary, and antepartum fetal surveillance, can reduce the maternal perinatal morbidity and mortality associated with the condition [18]. This is particularly important in developing countries where majority of women with diabetes prior to or during pregnancy are not diagnosed due to poor health seeking behaviour resulting from poverty and ignorance.

\section{Conclusion}

In conclusion, this study shows that the incidence of diabetes in pregnancy in Abakaliki is lower when compared to developed countries and the commonly recognized risk factors are valid for our women. Women with pregnancies complicated by diabetes had a higher incidence of adverse maternal and perinatal outcomes.

\section{Limitations}

The limitation of this study is that due to lack of follow up after discharge, the data on neonatal morbidity and mortality as well as maternal outcome for the rest of the puerperium were not available for analysis and finally retrospective nature of study limits its validity.

\section{Conflicts of Interest}

The authors declare no conflicts of interest regarding the publication of this paper.

\section{References}

[1] Hossein-Nezhad, A., Maghbooli, Z., Vassigh, A.R. and Larijani, B. (2007) Prevalence of Gestational Diabetes Mellitus and Pregnancy Outcomes in Iranian Women. Taiwanese Journal of Obstetrics \& Gynecology, 46, 236-241. https://doi.org/10.1016/S1028-4559(08)60026-1

[2] Clause, T.D., Mathiesen, E. and Ekbom, P. (2005) Poor Pregnancy Outcome in Women with Type 2 Diabetes. Diabetes Care, 28, 323-328. https://doi.org/10.2337/diacare.28.2.323

[3] Shingala, K.D., Shah, S.R., Vyas, R.C. and Parikh, P.M. (2019) Fetomaternal Outcome in Patients with Diabetes Mellitus in Pregnancy. International Journal of Reproduction, Contraception, Obstetrics and Gynecology, 8, 2701-2704. https://doi.org/10.18203/2320-1770.ijrcog20193028

[4] Fareed, P., Siraj, F. and Lone, K. (2017) Fetomaternal Outcome in Women with Gestational Diabetes Mellitus. International Journal of Research in Medical Sciences, 5, 4151-4154. https://doi.org/10.18203/2320-6012.ijrms20174001

[5] Metzger, B.E., Lowe, L.P., Dyer, A.R., Trimble, E.R., Chaovarindr, U., Coustan, D.R., et al. (2008) Hyperglycemia and Adverse Pregnancy Outcomes. The New England Journal of Medicine, 358, 1991-2002. https://doi.org/10.1056/NEJMoa0707943

[6] Saxena, P., Tyagi, S., Prakash, A., Nigam, A. and Trivedi, S.S. (2011) Pregnancy Outcome of Women with Gestational Diabetes in a Tertiary Level Hospital of North India. Indian Journal of Community Medicine, 36, 120-123. 
https://doi.org/10.4103/0970-0218.84130

[7] Casson, I.F., Clarke, C.A., Howard, C.V., McKendrick, O., Pennycook, S., Pharoah, P.O.D., et al. (1997) Outcomes of Pregnancy in Insulin Dependent Diabetic Women: Results of a Five Year Population Cohort Study. BMJ, 315, 275-278. https://doi.org/10.1136/bmj.315.7103.275

[8] Palatnik, A., Mele, L., Landon, M.B., et al. (2015) Timing of Treatment Initiation for Mild Gestational Diabetes Mellitus and Perinatal Outcomes. American Journal of Obstetrics \& Gynecology, 213, e1-e8. https://doi.org/10.1016/j.ajog.2014.10.814

[9] Black, M.H., Sacks, D.A., Xiang, A.H., et al. (2013) The Relative Contribution of Pre-Pregnancy Overweight and Obesity, Gestational Weight Gain, and IADPSG-Defined Gestational Diabetes Mellitus to Fetal Overgrowth. Diabetes Care, 36, 56-62. https://doi.org/10.2337/dc12-0741

[10] The International Federation of Gynecology and Obstetrics (FIGO) (2015) Initiative on Gestational Diabetes Mellitus: A Pragmatic Guide for Diagnosis, Management, and Care.

[11] Usami, T., Yokoyama, M., Ueno, M., Iwama, N., Sagawa, N., Kawano, R., et al. (2019) Comparison of Pregnancy Outcomes between Women with Early-Onset and Late-Onset Gestational Diabetes in a Retrospective Multi-Institutional Study in Japan. Journal of Diabetes Investigation. https://doi.org/10.1111/jdi.13101

[12] Orru, M.I., Nwose, E.U., Bwititi, P.T. and Igumbor, E.O. (2018) Screening for Gestational Diabetes: Evaluation of Prevalence in Age Stratified Subgroups at Central Hospital Warri Nigeria. International Journal of Reproduction, Contraception, $\mathrm{Ob}$ stetrics and Gynecology, 7, 63-68.

https://doi.org/10.18203/2320-1770.ijrcog20175487

[13] Mapira, H.T., Tumusiime, D.K., Yarasheski, K., Rujeni, N., Cade, T.W. and Mutimura, E. (2017) Strategy to Improve the Burden of Gestational Diabetes in African Women: Rwandan Perspective. Rwanda Journal, 4, 36.

https://doi.org/10.4314/rj.v4i1.5F

https://www.ajol.info/index.php/rj/article/view/156410

[14] Olagbuji, B.N., Atiba, A.S., Olofinbiyi, B.A., Akintayo, A.A., Awoleke, J.O., Ade-Ojo, I.P., et al. (2015) Prevalence of and Risk Factors for Gestational Diabetes Using 1999, 2013 WHO and IADPSG Criteria upon Implementation of a Universal One-Step Screening and Diagnostic Strategy in a Sub-Saharan African Population. The European Journal of Obstetrics \& Gynecology and Reproductive Biology, 189, 27-32. https://doi.org/10.1016/j.ejogrb.2015.02.030

[15] Macaulay, S., Dunger, D.B. and Norris, S.A. (2014) Gestational Diabetes Mellitus in Africa: A Systematic Review. PLoS ONE, 9, e97871. https://doi.org/10.1371/journal.pone.0097871

[16] Macaulay, S., Ngobeni, M., Dunger, D.B. and Norris, S.A. (2018) The Prevalence of Gestational Diabetes Mellitus amongst Black South African Women Is a Public Health Concern. Diabetes Research and Clinical Practice, 139, 278-287. https://doi.org/10.1016/j.diabres.2018.03.012

[17] Stogianni, A., Lendahls, L., Landin-Olsson, M. and Thunander, M. (2019) Obstetric and Perinatal Outcomes in Pregnancies Complicated by Diabetes, and Control Pregnancies, in Kronoberg, Sweden. BMC Pregnancy and Childbirth, 19, 159. https://doi.org/10.1186/s12884-019-2269-8

[18] Balsells, M., Garcia-Patterson, A., Gich, I. and Corcoy, R. (2009) Maternal and Fetal Outcome in Women with Type 2 versus Type 1 Diabetes Mellitus: A Systematic Review and Meta-Analysis. The Journal of Clinical Endocrinology \& Metabolism, 94, 4284-4291. https://doi.org/10.1210/jc.2009-1231 